\title{
Spectroscopic Diagnostics of Exploding Aluminum Wire Plasma in Distilled Water
}

\author{
Hassan Hashim \\ Department of Physics, College of Science, Al-Nahrain University, Baghdad-Iraq. \\ Corresponding Author: hassan.albattat@gmail.com.
}

\begin{abstract}
This work aims to study the exploding aluminum wire plasma parameters by using optical emission spectroscopy method. The emission spectra of the aluminum discharge plasma have been recorded and analyzed. The plasma electron temperature $\left(\mathrm{T}_{\mathrm{e}}\right)$ was calculated by Boltzmann plot, and the electron density $\left(\mathrm{n}_{\mathrm{e}}\right)$, by Stark broadening for aluminum wire of diameter $0.3 \mathrm{~mm}$ and different current 75,100 , and $160 \mathrm{~A}$ in distilled water. The aluminum line $656.279 \mathrm{~nm}$ was used to calculate the electron density by Stark broadening. It was found that the electron density $\left(\mathrm{n}_{\mathrm{e}}\right)$ increase from $28 \times 10^{16} \mathrm{~cm}^{-3}$ to $60 \times 10^{16} \mathrm{~cm}^{-3}$ with increasing current from 75 to $160 \mathrm{~A}$, while the electron temperatures increase from 0.986 to $1.614 \mathrm{eV}$ for the same conditions. The optical emission spectrum (OES) emitted from the plasma have a peak located at $653 \mathrm{~nm}$ corresponding to $\mathrm{H}_{\alpha}$ line for hydrogen atoms, and others peaks belong to Aluminum (AlI and AlII lines), and Oxygen (OI and OII lines). The relationship between the plasma electron temperature, emission line intensity and number density were studied. [DOI: 10.22401/JNUS.21.2.13]
\end{abstract}

Keywords: exploding wire; spectroscopy; Boltzmann plot; plasma characteristics.

\section{Introduction}

Optical emission spectroscopy is one of plasma diagnostics method. It is used to obtain information about the nature of plasma, such as the chemical compositions and plasma electron density and electron temperature [3]. Emission spectrum often consists of a number of characteristic atomic or ionic spectral lines [4].

The spectral line intensity $\left(\mathrm{I}_{\mathrm{ji}}\right)$ can be described as[3]:

$I_{j i}=\frac{N}{U(T)} g_{j} A_{j i} h v_{j i} e^{-E_{j} / k_{\beta} T_{e}}$

where $g_{j}$ is the density of states, $\mathrm{E}_{\mathrm{j}}$ is the upper level energy, $A_{j i}$ transition probability between the transition states of upper level (j) and lower level (i) and $\mathrm{T}_{e}$ is the electron temperature. Thus the electron temperature of plasma can be calculated using Boltzmann relation [5]:

$\ln \left(\frac{I_{j i} \lambda_{j i}}{g_{j} A_{j i}}\right)=\left(-\frac{E_{j}}{K T_{e}}\right)+\left(\frac{N(T)}{U(T)}\right)$

where $\lambda \mathrm{ji}$, is the wavelength corresponding to the transmission between level $\mathrm{j}$ and level $\mathrm{i}$. While, the electron density can be calculated, utilizing stark broadening relation [6]
$n_{e}\left(\mathrm{~cm}^{-3}\right)=\left[\frac{\Delta \lambda}{2 \omega_{s}\left(\lambda, T_{e}\right)}\right] N_{r}$

Whereas, $\Delta \lambda$ is the FWHM of the line, and $\omega_{\mathrm{s}}$ is the Stark broadening parameter, that can be found in the standard tables $N_{r}$ is the reference electron density which equal to $10^{16} \mathrm{~cm}^{-3}$ for neural atoms and $10^{17} \mathrm{~cm}^{-3}$ for singly charged ions. There are many parameters used to characterize the plasma such as Debye length $\left(\lambda_{\mathrm{D}}\right)$ which calculated as follows [7]:

$\lambda_{D}=\left(\frac{k_{\beta} T_{e}}{4 \pi e^{2} n_{0 e}}\right)^{1 / 2}$

Plasma frequency which calculated as follows [8].

$\omega_{p}=\sqrt{\frac{N_{e} q_{e}^{2}}{\epsilon_{o} m_{e}}}$

The number of particles in a Debye sphere $\left(\mathrm{N}_{\mathrm{D}}\right)[8]$

$N_{D}=n_{e}\left[\frac{4 \pi \lambda_{D}^{3}}{3}\right]=\frac{1.38 \times 10^{6} T_{e}^{3 / 2}}{n_{e}^{1 / 2}}$

where $T_{e}$ in $\mathrm{K}$

Plasma was produced by different techniques one of them is wire explosion, which has recently gained high attention [1]. Plasma formed by exploding wires using high 
electric energy pass throw the wire within short time in different ambient. Several parameters have a control in exploding wires technique such as voltage, current, material type, wire dimension, and the medium in which the explosion is performed, [2].

\section{Experimental part}

The explosion of wire is characterized by the energy introduced into the wire, which is higher than the evaporation energy of the material. The energy input time is shorter than the time required for the current to spread into the wire. The basic circuit for exploding the aluminum wires used in produce plasm is shown in Fig.(1). Aluminum wire with diametr of $0.3 \mathrm{~mm}$ and $20 \mathrm{~mm}$ long is used to produce aluminum plasma in $100 \mathrm{ml}$ distilled water. The emitted spectrum produced by the exploding aluminum wire was carried by optical fiber to be analyzed using a spectrometer connected with a computer to record the data, which then used to study the effect of the current on the properties of the produced plasma.

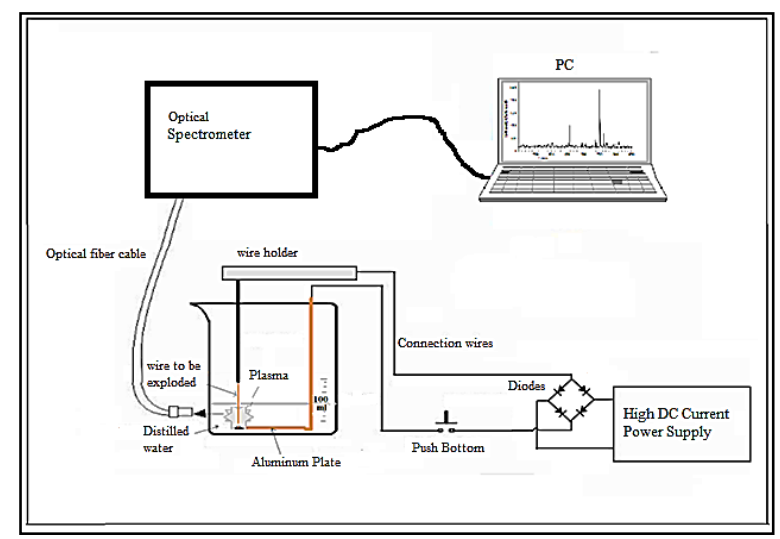

Fig.(1): Schematic diagram for the basic circuit used for the generation and characterization of the plasma.

\section{Results and Discussion}

Fig.(2) shows the optical emission spectrum (OES) emitted from the Plasma produced by exploding aluminum wire with diameter of $0.3 \mathrm{~mm}$ and different current 75,100 and $160 \mathrm{~A}$. It is noted from the figure that the peak located at $656 \mathrm{~nm}$ corresponding to $\mathrm{H}_{\alpha}$ line for hydrogen atoms produced from water molecular dissociate. The others peaks belong to AlI, AlII, OI and OII as the index to each of them. It can be noticed that the peaks intensities increase with increasing the current this result is in agreement with Wankhede (2013)[1].

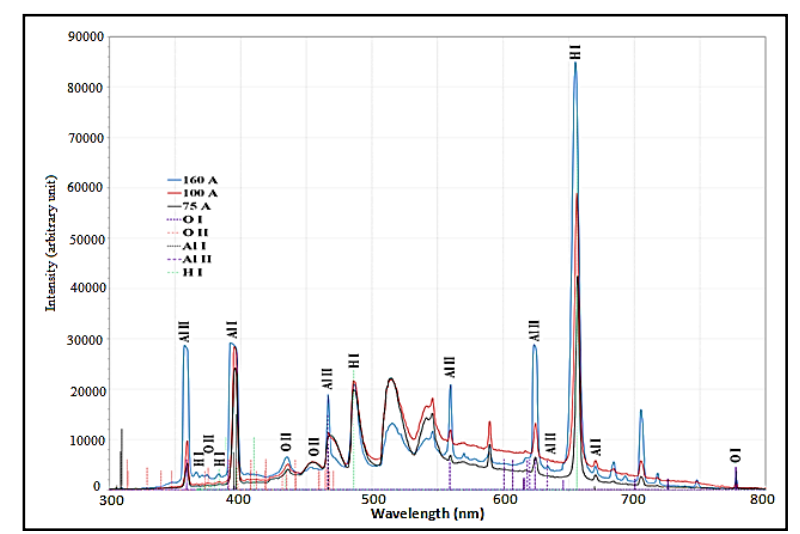

Fig.(2): Emission spectra for Aluminum exploding wire at different current 75,100 and 160A.

The values of electron temperature $\left(T_{e}\right)$ were calculated by Boltzmann plot using four of AlI lines at $(359.28,468.56,559.88$ and $634.55 \mathrm{~nm}$ ) for different currents, as shown in Fig.(3). The $T_{e}$ values were deduced from reveres of best fitting line for the relation between $\left(\ln \left(\frac{I_{j i} \lambda_{j i}}{g_{j} A_{j i}}\right)\right)$ versus upper energy level $\left(E_{j}\right)$.The constants values for the aluminum emission lines taken from Sansonetti J. E. and Martin W. C. (2005) [9]. The equations of fitting lines and the $\mathrm{R}^{2}$ were shown in Fig.(3), where $\mathrm{R}^{2}$ is the statistical coefficient indicating the goodness of the linear fit. 


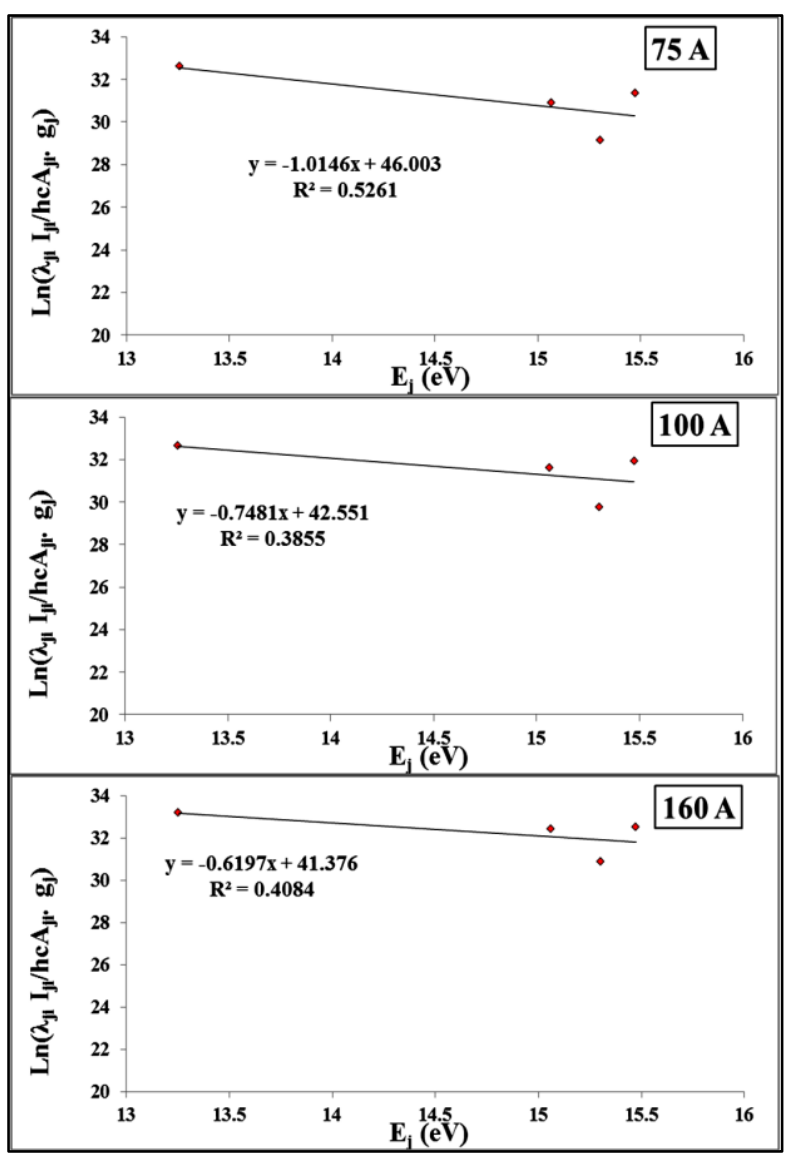

Fig.(3): Boltzmann plot from the Al I lines produced by exploding wire with different current in distilled water and the wire diameter of foro. $3 \mathrm{~mm}$.

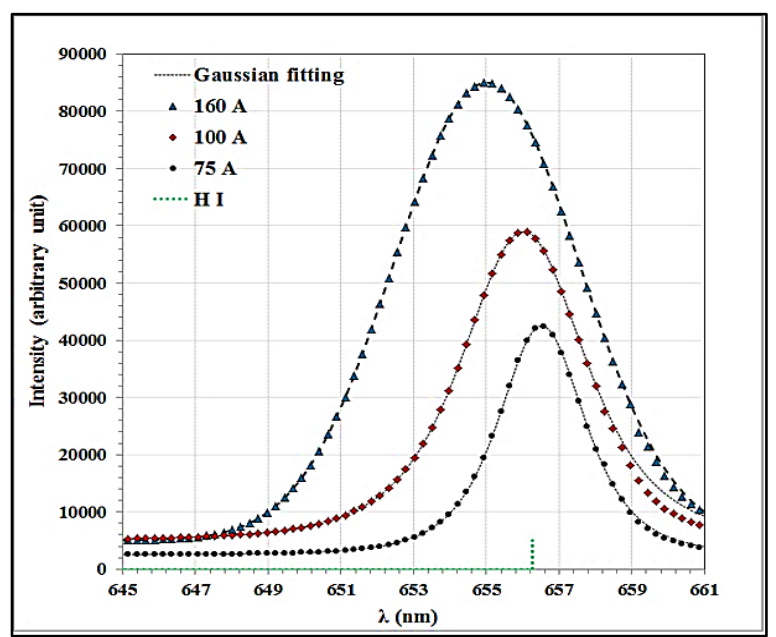

Fig.(4): Ho peaks at $656.279 \mathrm{~nm}$ broadening and there Gaussian fitting using different current.

Fig.(4) shows the aluminum line peak profile at wavelength $656.279 \mathrm{~nm}$. Where full width at half maximum found by using Gaussian fitting to calculate electron density for different samples using Stark effect depending on the standard values of broadening for this line [11]. It can be seen that the full width increase with current increasing, which indicate to increasing in electron density.

Fig.(5) shows the variation of electron temperature $\left(\mathrm{T}_{\mathrm{e}}\right)$, calculated by Boltzmann plot, and electron density $\left(\mathrm{n}_{\mathrm{e}}\right)$, using Stark broadening effect, with different current. This figure shows that the $n_{\mathrm{e}}$ increase from $28 \times 10^{16}$ to $60 \times 10^{16} \mathrm{~cm}^{-3}$ with increasing current from 75 to 160 A while the electron temperatures increase from 0.986 to $1.614 \mathrm{eV}$ for the seame conditions. This result is agreed with [1]. The decrement in concentration cause decreasing in collision which caused to increase in electron temperature, where the electron temperature loses by different ways (elastic, excitation and ionization collisions).

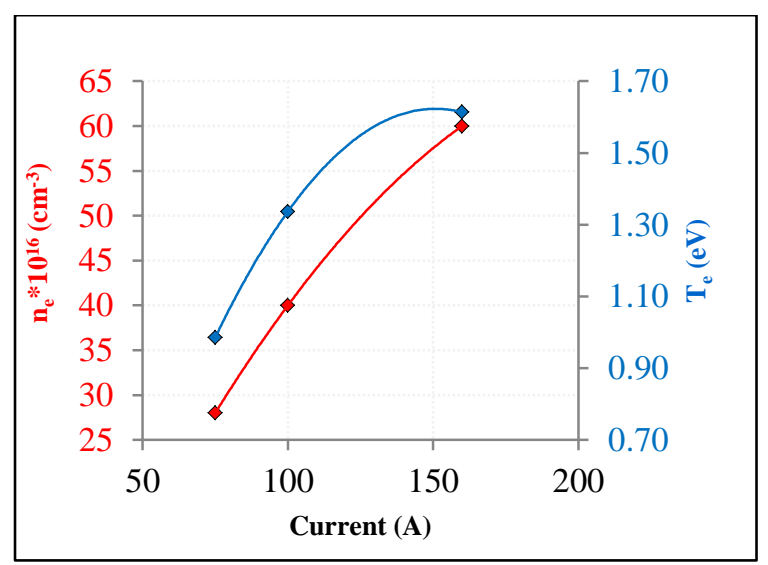

Fig.(5): Electron temperature $T_{e}$ and electron density $n_{e}$ for plasm produced by aluminum exploding wire with different current.

Table (1) show the calculated values of Debye length $\left(\lambda_{D}\right)$, plasma frequency $\left(f_{p}\right)$ and Debye number $\left(\mathrm{N}_{\mathrm{D}}\right)$ from aluminum plasma produced by exploding wire with different with diameters current 57,100,160A. 
Table (1)

Plasma parameters calculated from spectroscopy lines for aluminum exploding wire with different current 57,100 and 160A.

\begin{tabular}{|c|c|c|c|c|c|c|}
\hline $\begin{array}{c}\text { Current } \\
\text { (A) } \\
\end{array}$ & $\begin{array}{c}\mathbf{T}_{\mathrm{e}} \\
(\mathrm{eV})\end{array}$ & FWHM (nm) & $n_{e} * 10^{16}\left(\mathrm{~cm}^{-3}\right)$ & $f_{p}(H z) * 10^{12}$ & $\lambda_{\mathrm{D}} * 10^{-5}(\mathrm{~cm})$ & $\mathbf{N}_{D} * 10^{3}$ \\
\hline 75 & 0.986 & 2.80 & 28.000 & 4.752 & 1.394 & 3.177 \\
\hline 100 & 1.337 & 4.00 & 40.000 & 5.679 & 1.353 & 4.148 \\
\hline 160 & 1.614 & 6.00 & 60.000 & 6.956 & 1.214 & 4.492 \\
\hline
\end{tabular}

\section{Conclusions}

Study the effect of aluminum wire diameter in exploding wire technique on spectra for produced emissions and the plasma characteristics shows many points as follows:

- Many atomic and ionic aluminum peaks, and a strong peak corresponding to $\mathrm{H}_{\alpha}$ line appeared in all spectra.

- The peaks intensities increase with increasing the current, as a result of increasing the input energy.

- The electron density and electron temperature increase with increasing current.

\section{References}

[1] Wankhede P., Sharma P. K., and Jha A. K., "Synthesis of Copper Nanoparticles through Wire Explosion Route," J. Eng. Res.and Appl., 3(6), 1664-1669, 2013.

[2] Alqudami A. and Annapoorni S., Shivaprasad S.M., "Ag-Au alloy nanoparticles prepared by electro-exploding wire technique," J Nanopart Res, 10(6), 1027-1036, 2008.

[3] Devia D. M., Rodriguez-Restrepo L. V, and Restrepo-Parra E., "Methods Employed in Optical Emission Spectroscopy Analysis," Ing. Cienc., 11(21), 239-267, 2015.

[4] Zhukov M. F., Ovsyannikov A. A., Plasma Diagnostics, Ch. 5. UK: Cambridge International Science Publishing, 2005.

[5] Hamed S. S., "Spectroscopic Determination of Excitation Temperature and Electron Density in Premixed Laminar Flame," Egypt J. Solids, 28(2), 349-357, 2005.

[6] Shaikh N. M., Hafeez S., Rashid B., and Baig M. A., "Spectroscopic studies of laser induced aluminum plasma using fundamental, second and third harmonics of a Nd: YAG laser", Eur. Phys. J ournal D, 44(2), 371-379, 2007.

[7] Eliezer S., "The Interaction of High-Power Lasers With Plasmas," Plasma Phys. Control. Fusion, 45(2), 181-181, 2003.

[8] Inan,U. S. and Golkowski, M., Principles of Plasma Physics for Engineers and Scientists, Vol. 1. New York: Cambridge University Press, 2011.

[9] Sansonetti J. E. and Martin W. C., "Handbook of Basic Atomic Spectroscopic Data," Am. Inst. Phys., 34(4),1559-2259, 2005.

[10] Parigger C. and Lewis J., "Measurements of sodium chloride concentration in water droplets using laser-induced plasma spectroscopy" Appl. Phys., 12(1), 163-173, 1993.

[11] Konjevic N., Lesage A., Fuhr J. R., and Wiese W. L., "Experimental Stark Widths and Shifts for Spectral Lines of Neutral and Ionized Atoms (A Critical Review of Selected Data for the Period 1989 Through 2000)", J. Phys. Chem. Ref. Data, 31(3), 819-926, 2002. 